\title{
Assessment of Periodontal Status in Patients with Dental Fluorosis - A Retrospective Hospital Based Study
}

\author{
R. Saravanan, Priya Lochana Gajendran* and Balaji Ganesh S \\ Saveetha Dental College and Hospitals, Saveetha Institute of Medical and Technical Science, \\ Saveetha University, 162, Poonamaelle High Road Chennai. India \\ Corresponding author email: priya.sde@saveetha.com
}

\begin{abstract}
Dental fluorosis is a defect caused in the enamel of the tooth structure due to more fluoride intake during the developmental stages of teeth. The effect of fluoride on the periodontal tissues remains obscure. Thus, the aim of the study was to retrospectively assess the periodontal status of patients with dental fluorosis who had reported to a private dental hospital. A total of 378 patients who had dental fluorosis were included in the study. Periodontal status, Dean's fluorosis index, oral hygiene index values for all the patients were recorded. The required data was collected and SPSS Software was used to assess the data. Among the study population, $71 \%$ of them were males and $29 \%$ of them were females. According to the Deans fluorosis index, among the study population, a total of $1.6 \%$ of subjects had a score of questionable, $21 \%$ of subjects had very mild, $37 \%$ of subjects has mild fluorosis, $31 \%$ had moderate and around $9 \%$ of the patients had severe form of fluorosis. There was no statistically significant association seen between age and the degree of dental fluorosis ( $\mathrm{p}$ value 0.602 ). The association between Dean's fluorosis index and periodontal status was found to be statistically not significant. ( $\mathrm{p}$ value-0.300). The severity of dental fluorosis was more in the gingivitis patients than periodontitis patients, however the results were not statistically significant. Within the limitations of the present retrospective analysis, a possible relationship exists between periodontal disease and degree of dental fluorosis with a male prevalence for dental fluorosis. A mild to moderate degree of dental fluorosis was seen in the majority of the study subjects. The severity of dental fluorosis was more in the gingivitis patients than periodontitis patients, however the results were not statistically significant.
\end{abstract}

\section{KEY WORDS: FLUOROSIS; GINGIVITIS; ORAL HYGIENE INDEX; PERIODONTITIS.}

\section{INTRODUCTION}

Periodontitis is a chronic inflammatory disease resulting in the loss of supporting tissues of the teeth. The increase in the various pro-inflammatory cytokines levels have been associated with the etiopathogenesis of the disease. Many local and systemic factors play a significant role in modulating the severity of periodontal disease. One such attributing factor is fluoride. "Dental fluorosis," a specific disturbance in tooth formation and an esthetic condition, is defined as a chronic, fluoride-induced condition, in which enamel development is disrupted and the enamel is hypo mineralized. The dental fluorosis is mainly associated with the defects in the formation of enamel during its

Biosc Biotech Res Comm P-ISSN: 0974-6455 E-ISSN: 2321-4007

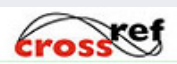

Identifiers and Pagination

Year: 2021 Vol: 14 No (10) Special Issue

Pages: 115-121

This is an open access article under Creative

Commons License Attribn 4.0 Intl (CC-BY).

DOI: $h t t p: / / d x . d o i . o r g / 10.21786 / b b r c / 14.10 .19$ developmental stage. The severity of the porosity on the enamel surface is highly dependent on the concentration of the fluoride in the tissue fluids during tooth formation. Clinically fluorosis appears as whitish spots, or opaque lines or as some striations on the surface of teeth. The brown stains are evident in cases of moderate to severe fluorosis, due to the uptake of extrinsic stains mainly from the diet. The severity of fluorosis also varies from one tooth to another depending on its time of eruption in the oral cavity (Zimmermann et al., 1955; Englander et al.,1963; DenBesten et al., 2011; Khalid, 2017; Varghese et al., 2015; Mootha et al., 2016; Khalid et al., 2016; Singh et al., 2018).

The effect of fluoride on dental caries has been well established. Various human studies which have analysed the effect of elevated fluoride in drinking water on gingivitis and periodontitis have shown inconsistent results. The effects of fluoride on periodontal tissues have also been controversial, Studies to assess the periodontal status in fluorosis subjects have been done in few parts of the country. Vandhana et al conducted a study at high fluoride belts of Davangere District, Karnataka State, India, they showed a strong association between periodontal diseases with high
Article Information

Received: $18^{\text {th }}$ Aug 2021

Accepted after revision: $25^{\text {th }}$ Oct 2021 
fluoride water in a population of 15-74 years (Massler et al 1951; Drummond et al., 1993; Grembowski et al., 1993; Bergstrom, 2003; Vandana et al., 2007; Molina-Frechero et al., 2012; Chopra et al 2016; Pritma et al 2020).

Previously our team has a rich experience in working on various research projects across multiple disciplines (Gheena and Ezhilarasan, 2019; Ke et al., 2019; Malli et al., 2019; Mehta et al., 2019; Samuel et al., 2019; Sharma et al., 2019; Vignesh et al., 2019; Jain et al., 2019; Jose et al., 2020; Krishnaswamy et al., 2020; Muthukrishnan et al., 2020; Samuel et al., 2020; Sathish and Karthick, 2020). Now the growing trend in this area motivated us to pursue this project. Pritma et al clearly stated that although the effect of fluoride on caries has been extensively discussed but the effect of fluorosis on the periodontium yet remains unclear (Pritma et al 2020). The exact mechanism and the relationship between dental fluorosis and periodontal disease remains obscure, thus the present study aims to assess the periodontal status of dental fluorosis patients retrospectively.

\section{MATERIAL AND METHODS}

The retrospective study was carried out by the analysis of the patients records from June 2019-March 2020. The study design was reviewed and approved by the ethical committee of Saveetha Institute of Medical and Technical Sciences (SIMATS). Prior permission to utilize the data from the university and ethical board number was obtained from the institution. The data from 378 patients who were diagnosed with Dental Fluorosis at SIMATS were included in this study. Case sheets with incomplete data were excluded from the study. Demographic details such as patient identity number (PID. No), age and sex were recorded. Periodontal parameters such as bleeding on probing, probing pocket depth, clinical attachment level was obtained from the patient records.

The oral hygiene status was assessed using the simplified - oral hygiene index (Greene and Vermillion 1964). The severity of fluorosis was assessed by Dean's fluorosis index (Trendley Dean 1942). Patients with Aggressive periodontitis, smoking, those with previous history of periodontal surgery, and presence of any other systemic disease were excluded from the study. Based on the severity of the periodontal disease the patients were categorised into those who had generalised chronic gingivitis, patients with localised chronic periodontitis and patients with generalised chronic periodontitis. The data obtained analysed using statistical software SPSS version 23.0 (Statistical Package for The Social Sciences). Chi-square test was used to study association between the parameters. The results were considered to be of statistical significance if $\mathrm{p}<0.05$.

\section{RESULTS AND DISCUSSION}

A total of 378 patients with dental fluorosis was included in this retrospective analysis. Majority of the subjects (84\%) were in the age group of 14 to 34 years, whereas the remaining $15 \%$ of patients were in the age group of 35 to 50 years and only $1 \%$ of the subjects were within the age group of 50- 55 years (Figure 1). Among the study population, $71 \%$ of them were males and $29 \%$ of them were females (Figure 2). The oral hygiene status of the study subjects was analysed using the $\mathrm{OHI}$ index. $45 \%$ of patients had good oral hygiene, $47 \%$ of patients had a score of fair and the remaining $8 \%$ of patients had poor oral hygiene (Figure 3). Based on the periodontal diagnosis, the majority ( $73 \%)$ of the subjects had generalised chronic gingivitis, 20 $\%$ had localised chronic periodontitis and the remaining $7 \%$ of patients were diagnosed with generalised chronic periodontitis (Figure 4).

Figure 1: This graph represents the percentage distribution of different age groups of the study subjects, where $X$ axis denotes age and $Y$ axis denotes number of patients. Grey colour denotes age distribution of 14-34 years of age, Blue colour denotes age distribution of 35-55 years of age and Green colour denotes age distribution of 55-80 years of age. Majority of patients are in the age group of 14-34 years of age $(83.86 \%)$ followed by $35-55$ years of age $(14.81 \%)$ and $55-80$ years of age $(1.32 \%)$.

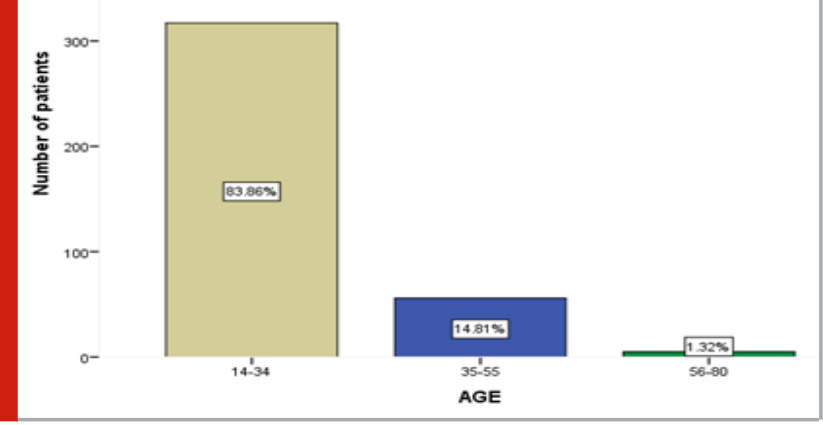

Figure 2: This graph represents the percentage of gender distribution among the study population, where $\mathrm{X}$ axis denotes Gender criteria and $Y$ axis denotes number of patients. Grey colour denotes Male and Blue colour denotes Female. There were more number of male subjects $\mathbf{( 7 1 . 4 3 \% )}$ than females $(28.57 \%)$.

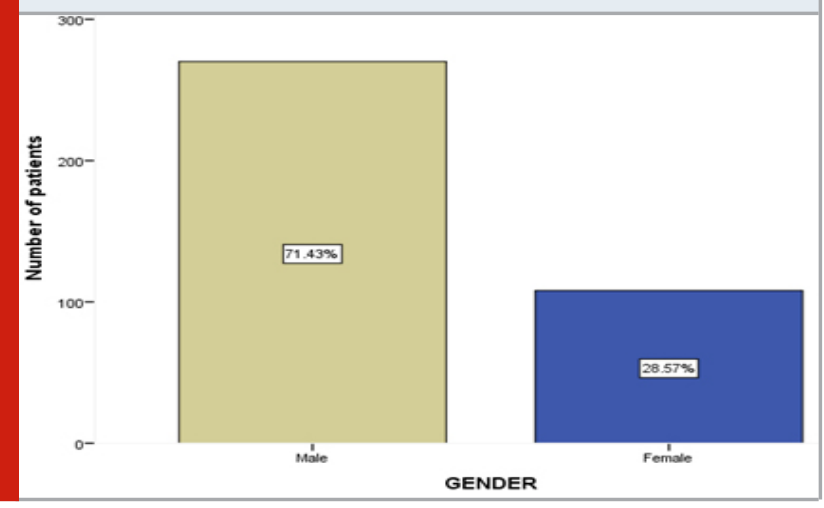

According to the Deans fluorosis index, among the study population, only $1.6 \%$ of subjects had a score of questionable, $21 \%$ of subjects had very mild,37\% of subjects had mild Fluorosis, 31\%had moderate fluorosis and around $9 \%$ of the patients had severe form of fluorosis 
(Figure 5). The association between age and periodontal status was found to be statistically significant. ( $p$ value0.000 ) Thus patients in the younger age group (14-34 years) had predominantly generalised chronic gingivitis whereas the older age group (55-80 years) had generalised chronic periodontitis (Figure 6).

Figure 3: This graph represents Oral hygiene index score values among the study population, where $X$ axis denotes oral hygiene index and $Y$ axis denotes total number of patients. Grey colour denotes Good, Blue colour denotes Fair and Green colour denotes Poor. The oral hygiene status was good in $\mathbf{4 5 . 7 7 \%}$ of patients, fair in $47.35 \%$ of patients, whereas only a smaller percentage of $6.88 \%$ had poor oral hygiene.
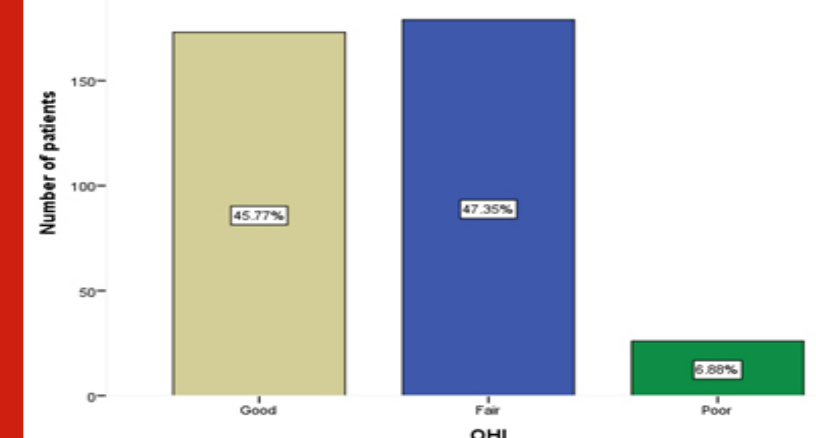

$\mathrm{OHI}$

Figure 4: This graph represents Periodontal status of the study subjects, where $X$ axis denotes Periodontal diagnosis and $\mathrm{Y}$ axis denotes number of patients. Grey colour denotes Generalised chronic gingivitis, Blue colour denotes Generalised chronic gingivitis with localised chronic Periodontitis and Green colour denotes Generalised chronic Periodontitis. Generalised chronic gingivitis $\mathbf{( 7 3 . 8 1 \% )}$ was predominantly seen among the subjects with lesser percentage of Generalised chronic gingivitis with localised chronic Periodontitis (20.63\%) and Generalised chronic Periodontitis $(5.56 \%)$

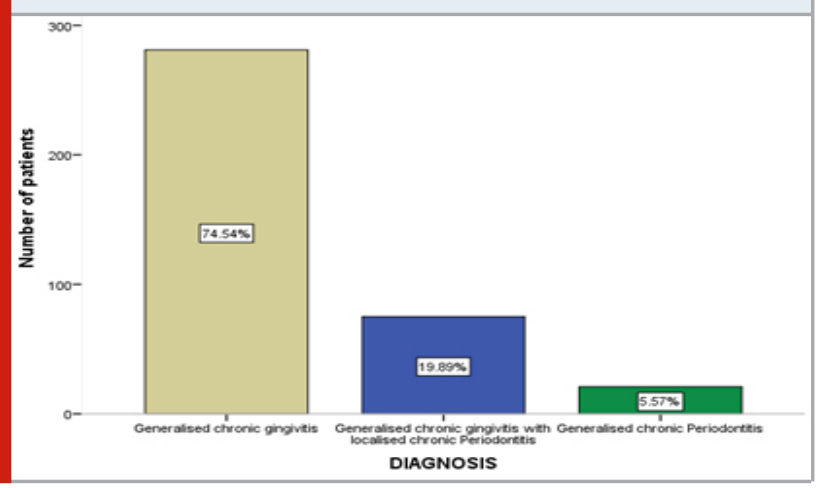

There was no association seen between age and the degree of dental fluorosis. ( $p$ value 0.602). However, people in the age group of 14-34 years were predominantly diagnosed with dental Fluorosis when compared with the other age groups (Figure 7). The association was found between Dean's fluorosis index and oral hygiene index was not statistically significant ( $\mathrm{p}$ value-0.051). The subjects with good oral hygiene had very mild forms of fluorosis followed by subjects with fair oral hygiene had moderate type of dental fluorosis and those who had poor oral hygiene had severe forms of dental fluorosis (Figure8).

Figure 5: This graph represents the distribution of Dean's Fluorosis index among the subjects, where $X$ axis denotes Dean's Fluorosis index and $Y$ axis denotes number of patients. Grey colour denotes Questionable, Blue colour denotes Very Mild, Green colour denotes Mild, Red colour denotes moderate and Orange denotes severe. From this figure we can infer that mild $(37.30 \%)$ and moderate $\mathbf{( 3 0 . 9 5 \% )}$ degrees of dental fluorosis were predominantly seen among the study subjects.

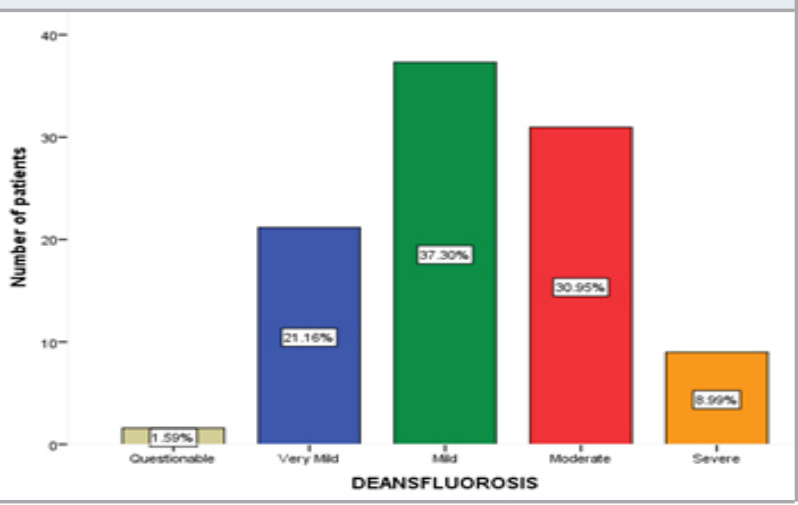

Figure 6: This bar graph depicts the relationship between age and periodontal status of the study population, where $\mathrm{X}$ axis denotes age groups and $\mathrm{Y}$ axis denotes the number of patients. Grey colour denotes Generalised chronic gingivitis, Blue colour denotes Generalised chronic gingivitis with localised chronic Periodontitis and Green colour denotes Generalised chronic Periodontitis. Chi square test was done and the association between age and periodontal status was found to be statistically significant. (Pearson's Chi square value: 43.33 , df -4 , p value- 0.000$)$ Thus patients in the younger age group (14-34 years) had predominantly generalised chronic gingivitis whereas the older age group (55-80 years) had generalised chronic periodontitis ( $p$ value less than 0.05).

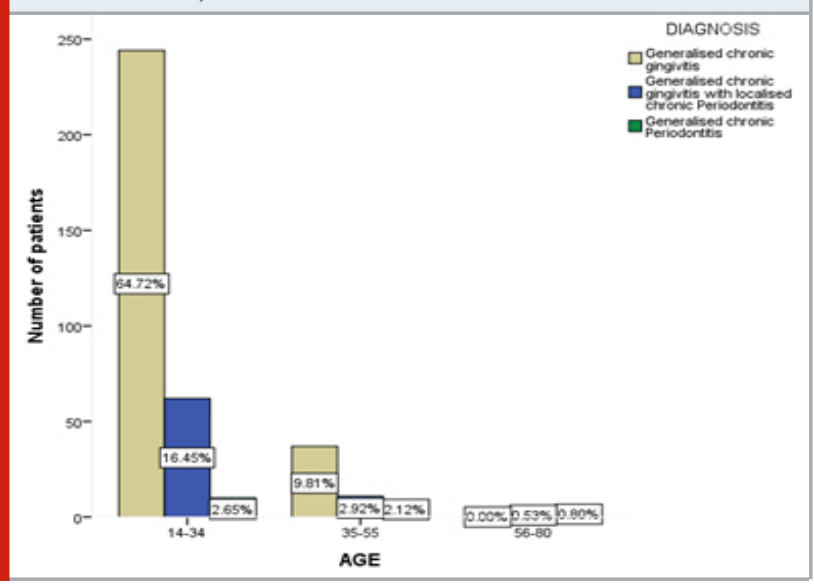


Figure 7: This graph represents the relationship between age and Dean's Fluorosis index among the subjects, where $X$ axis denotes age groups and $Y$ axis denotes number of patients. Grey colour denotes Questionable, Blue colour denotes Very Mild, Green colour denotes Mild, Red colour denotes Moderate and Orange colour denotes Severe. Chi square test was done and the association between age and dental fluorosis was found to be statistically not significant. (Pearson's Chi square value:6.408, df - 8, p value-0.602) Thus there is no association seen between age and the degree of dental fluorosis.(p value not less than 0.05). However, subjects in the age group of 14-34 years were predominantly diagnosed with dental Fluorosis when compared with the other age groups.

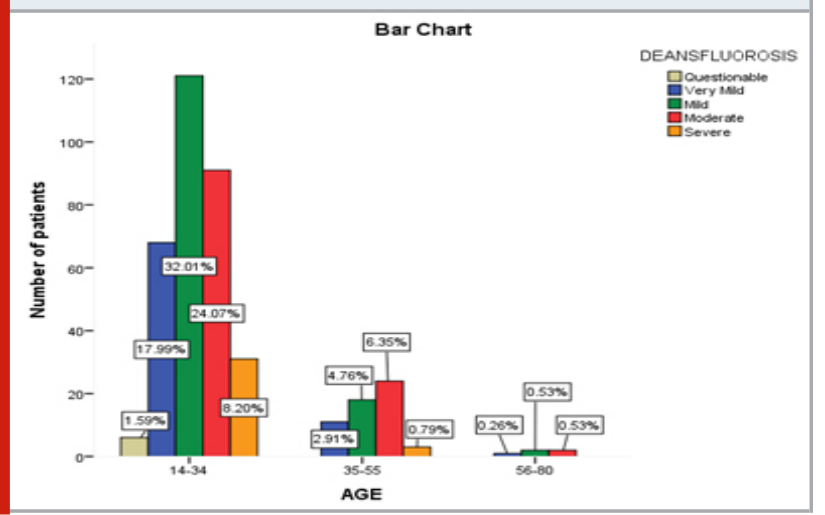

Figure 8: This graph represents the relationship between Dean's fluorosis index and Oral hygiene index (OHI) among the subjects, where $X$ axis denotes Oral hygiene index and $Y$ axis denotes number of patients. Grey colour denotes Questionable, Blue colour denotes Very Mild, Green colour denotes Mild, Red colour denotes Moderate and Orange colour denotes Severe. Chi square test was done and the association between Dean's fluorosis index and Oral hygiene index was not found to be statistically significant. (Pearson's Chi square value: 15.466 , df -8 , p value- 0.051 ). Majority of patients with dental fluorosis had good to fair oral hygiene index values and only a small percentage had poor oral hygiene maintenance, however the results were not statistically significant. ( $p$ value not less than 0.05 )

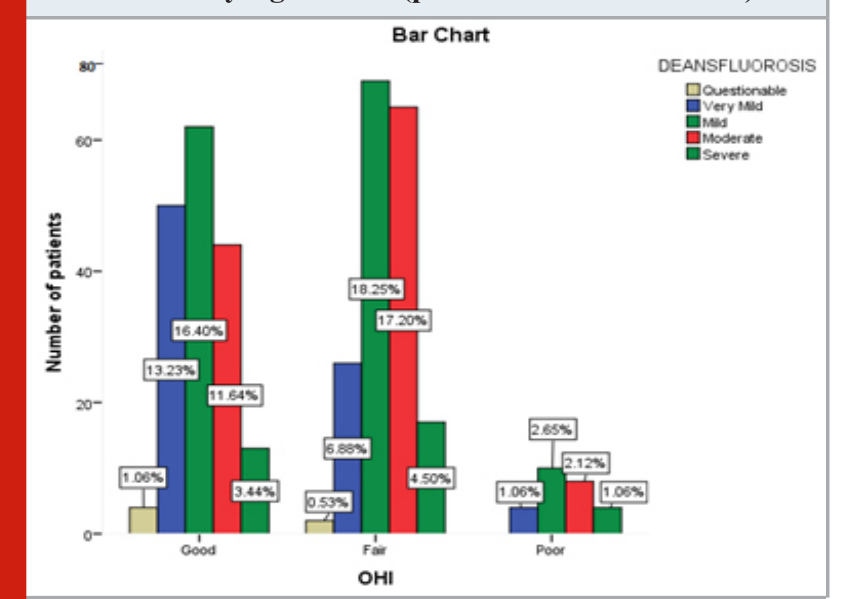

Figure 9: This graph represents the relationship between Dean's fluorosis index and periodontal status among the subjects, where $X$ axis denotes periodontal status and $Y$ axis denotes number of patients. Grey colour denotes Questionable, Blue colour denotes Very Mild, Green colour denotes Mild, Red colour denotes Moderate and Orange colour denotes Severe. Chi square test was done and the association between Dean's fluorosis index and periodontal status was found to be not statistically significant (Pearson's Chi square value:9.520, df - 8 , $p$ value- 0.300 ). Thus, the severity of dental fluorosis was more in the gingivitis patients than periodontitis patients however, the results were not statistically significant. ( $p$ value not less than 0.05 )

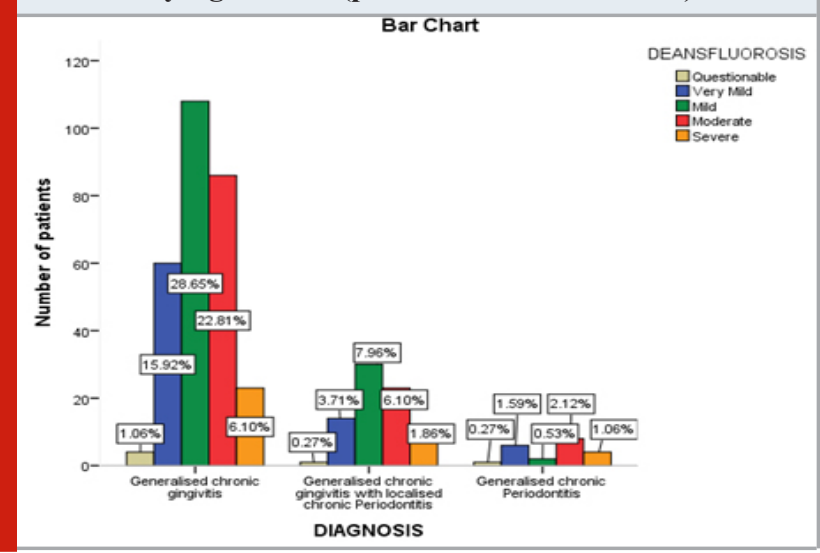

The association between Dean's fluorosis index and periodontal status was found to be statistically not significant.(p value- 0.300$)$. The severity of dental fluorosis was more in the gingivitis patients than periodontitis patients, however the results were not statistically significant (Figure 9).

In the present study the prevalence of dental fluorosis was higher in males $(71 \%)$ than females. This was in accordance with the study done by Vandhana et al and Kumar et al. In the present study majority of the study subjects had only gingivitis $(73 \%)$, similar observations were concluded by few other authors. The prevalence of periodontitis was low in the present study, with about $20 \%$ of subjects having localised chronic periodontitis and the only $7 \%$ of patients had generalised chronic periodontitis. Our findings are similar to the study done by Kumar et al with an overall lesser prevalence (12.3\%) of periodontitis in dental fluorosis patients (Haikel et al., 1989; Kumar et al., 2007; Vandana et al., 2007; Kumar et al., 2007; Vandana and Sesha Reddy, 2007; Chopra et al 2016; Pritma et al 2020).

Age is considered to be an important risk factor for periodontitis. In the present study, the majority of the patients who had fluorosis were in the age group of 14-34 years. Drummond et al conducted a study on the age group of 15-65 years, found that gingivitis and gingival recession to be more prevalent in high-fluoride areas than low-fluoride areas. Grembowski et al suggested that fluoridation has minimal beneficial effects on periodontal health when compared with adults who had no exposure. In the present study both males and females had been affected with 
gingivitis and periodontitis. There was a male predilection for periodontitis than females, as observed by Kumar et al. However, Vandana et al reported that females predominantly had periodontitis than males. Murray et al found gingivitis to be more prevalent in high-fluoride areas despite good oral hygiene. (Murray et al., 1972; Drummond, 1993; (Grembowski et al., 1993; Kumar et al., 2007; Vandana et al., 2007; Chopra et al 2016; Pritma et al 2020).

Although the role of dental plaque as a causative factor remains undoubted, the susceptibility of patients to develop periodontitis depends on many factors. The effect of fluoride on the periodontal tissues remains controversial. Possible reasons for fluorine being a contributing factor for the development of periodontitis have been reported in few animal studies. An animal study on cattle revealed that fluoride intoxication resulted in hypercementosis. The toxic effect of fluoride results in cementum necrosis and also to cyst formation. Osteonecrosis of the alveolar bone and recession of gingiva has been observed due to fluoride intoxication. Gross examination of teeth with mottled enamel, revealed that the root surfaces were irregular, with heavy deposits of calcified marks in the form of excessive amounts of fluoride or osteo cementum at the apical region of the teeth. A scanning electron microscopic study of human fluorosed and non fluorosed teeth reported partial mineralization of connecting tissue fibers and also presence of globular mineralized deposits in the healthy fluorosed teeth (Maylin and Krook, 1982; Maylin and Krook, 1982; Singh et al., 1962; Singh et al., 2018).

When comparing the periodontal status with dental fluorosis, the results did not show a statistically significant association. This could be attributed to the smaller sample size of the study population. However, the severity of dental fluorosis was more in the gingivitis patients than periodontitis. Kumar et al concluded that the severity of periodontal disease decreased as the degree of fluorosis increased, suggesting that when the fluoride level in the drinking water ranges from 1.83 to $2.01 \mathrm{ppm}$, fluoride in water is beneficial to the periodontal tissues. On the contrary, Vandana et al found that as the degree of fluorosis increased, severity of gingivitis reduced and periodontitis increased, suggesting a strong association between dental fluorosis and periodontitis. Our institution is passionate about high quality evidence based research and has excelled in various fields (Vandana et al 2007; Kumar et al., 2007; Pc et al., 2018; Ramesh et al., 2018; Ezhilarasan et al., 2019; Ramadurai et al., 2019; Sridharan et al., 2019; Vijayashree, 2019; Mathew et al., 2020). We hope this study adds to this rich legacy.

Thus, from various literature evidence high-fluoride water levels can result in cemental necrosis, osteosclerosis and calcification of periodontal ligaments. Contrary to this, fluoride has some antibacterial effects on microorganisms and also has a role in the treatment of periodontal disease. Thus it still remains controversial whether fluorosis is a boon or bane to periodontitis. Further longitudinal studies assessing the effects of fluorosis on periodontitis patients is needed. The periodontal status of patients in various high fluoride different parts of India should be carried out in future. This might help understand the disease and aid its management as a large part of world including India millions are still affected with fluorosis (Massler and Schour, 1951; Ranade, 1981).

\section{CONCLUSION}

Within the limitations of the present retrospective analysis, a possible relationship exists between periodontal disease and degree of dental fluorosis. Based on our study observations, a male prevalence was seen and people in the age group of 14-34 years were predominantly diagnosed with dental fluorosis when compared with the other age groups. A mild to moderate degree of dental fluorosis was seen in the majority of the study subjects. The severity of dental fluorosis was more in the generalised chronic gingivitis patients than generalised chronic periodontitis patients, however the results were not statistically significant.

\section{REFERENCES}

Bergstrom, J. (2003). Tobacco smoking and risk for periodontal disease. Journal of Clinical Periodontology; pp. 107-113.

Chopra A, Kaur G, Lakhanpal M, Gupta N, Suri V, Bhudhiraja S (2016). A cross-sectional study to assess the effect of dental fluorosis on periodontal status. J Indian Assoc Public Health Dent; 14:150-3

DenBesten, P. and Li, W. (2011). Chronic Fluoride Toxicity: Dental Fluorosis, Fluoride and the Oral Environment; pp. 81-96.

Drummond, B. K. (1993). Fluorides in caries prevention, 3rd edition, Journal of Dentistry, p. 317.

Englander, H. R., Kesel, R. G. and Gupta, O. P (1963). The Aurora-Rockford, Ill., Study II. Effect of Natural Fluoride on the Periodontal Health of Adults. American Journal of Public Health and the Nations Health, pp. 1233-1242.

Ezhilarasan, D., Apoorva, V. S. and Ashok Vardhan, N (2019). Syzygium cumini extract induced reactive oxygen species-mediated apoptosis in human oral squamous carcinoma cells. Journal of oral pathology \& medicine; 48(2), pp. 115-121.

Gheena, S. and Ezhilarasan, D (2019). Syringic acid triggers reactive oxygen species-mediated cytotoxicity in HepG2 cells. Human \& experimental toxicology, 38(6), pp. 694-702.

Grembowski, D. et al. (1993). Fluoridation effects on periodontal disease among adults. Journal of periodontal research, 28(3), pp. 166-172.

Haikel, Y. et al. (1989). Periodontal treatment needs in populations of high- and low-fluoride areas of Morocco, Journal of Clinical Periodontology. pp. 596-600.

Jain, V. S. et al. (2019). Evaluation of Three-Dimensional Changes in Pharyngeal Airway Following Isolated Lefort One Osteotomy for the Correction of Vertical Maxillary Excess: A Prospective Study. Journal of maxillofacial and 
oral surgery, 18(1), pp. 139-146.

Jose, J., Ajitha and Subbaiyan, H. (2020). Different treatment modalities followed by dental practitioners for Ellis class 2 fracture - A questionnaire-based survey. The open dentistry journal, 14(1), pp. 59-65.

Ke, Y. et al. (2019). Photosynthesized gold nanoparticles from Catharanthus roseus induces caspase-mediated apoptosis in cervical cancer cells (HeLa), Artificial cells, nanomedicine, and biotechnology, 47(1), pp. 19381946.

Khalid, W. (2017). Comparison of Serum Levels of Endothelin-1 in Chronic Periodontitis Patients Before and After Treatment. Journal of Clinical and Daignostic Research 11(4): ZC78-ZC81.

Khalid, W. et al. (2016). Role of endothelin-1 in periodontal diseases: A structured review. Indian journal of dental research, 27(3), pp. 323-333.

Krishnaswamy, H. et al. (2020). Investigation of air conditioning temperature variation by modifying the structure of passenger car using computational fluid dynamics. Thermal science, 24(1 Part B), pp. 495-498.

Kumar, R. H. et al. (2007). Assessment of Current Status of Fluorosis in North-Western Districts of Tamil Nadu Using Community Index for Dental Fluorosis. Journal of Human Ecology, pp. 27-32.

Malli Sureshbabu, N. et al. (2019). Concentrated Growth Factors as an Ingenious Biomaterial in Regeneration of Bony Defects after Periapical Surgery: A Report of Two Cases. Case reports in dentistry; p. 7046203.

Massler, M. and Schour, I. (1951). Relation Of Malnutrition, Endemic Dental Fluorosis and Oral Hygiene to the Prevalence and Severity of Gingivitis, Journal of Periodontology, pp. 205-211.

Mathew, M. G. et al. (2020). Evaluation of adhesion of Streptococcus mutans, plaque accumulation on zirconia and stainless-steel crowns, and surrounding gingival inflammation in primary teeth, Clinical oral investigations. ;24(9):3275-3280

Maylin, G. A. and Krook, L. (1982). Milk production of cows exposed to industrial fluoride pollution. Journal of Toxicology and Environmental Health, pp. 473-478.

Mehta, M. et al. (2019). Oligonucleotide therapy: An emerging focus area for drug delivery in chronic inflammatory respiratory diseases. Chemico-biological interactions, 308, pp. 206-215.

Molina-Frechero, N. et al. (2012). Fluorosis and dental caries: an assessment of risk factors in Mexican children. Revista de investigacion clinica; organo del Hospital de Enfermedades de la Nutricion, 64(1), pp. 67-73.

Mootha, A. et al. (2016). The Effect of Periodontitis on Expression of Interleukin-21: A Systematic Review. International Journal of Inflammation, pp. 1-8.

Muthukrishnan, S. et al. (2020). Support vector machine for modelling and simulation of heat exchangers. Thermal science, 24(1 Part B), pp. 499-503.

Pc, J., Marimuthu, T. and Devadoss, P. (2018). Prevalence and measurement of anterior loop of the mandibular canal using CBCT: A cross sectional study, Clinical implant dentistry and related research. Aug;20(4):531-534

Pritma Singh, N.D. Gupta, Afshan Bey (2020)Dental fluorosis and periodontium: A game of shadows?J Oral Biol Craniofac Res. 2014 Jan-Apr; 4(1): 47-48.

Ramadurai, N. et al. (2019). Effectiveness of $2 \%$ Articaine as an anesthetic agent in children: randomized controlled trial, Clinical oral investigations, 23(9), pp. 3543-3550.

Ramesh, A. et al. (2018). Comparative estimation of sulfiredoxin levels between chronic periodontitis and healthy patients - A case-control study, Journal of periodontology, 89(10), pp. 1241-1248.

Ranade, S. N. (1981). Social and Economic Change in India: Studies in Rural Development: Dan A. Chekki, Ed: Participatory Democracy in Action: International Profiles of Community Development. Journal of International Affairs, pp. 317-318.

Samuel, M. S. et al. (2019). Efficient removal of Chromium (VI) from aqueous solution using chitosan grafted graphene oxide (CS-GO) nanocomposite. International journal of biological macromolecules, 121, pp. 285-292. Samuel, S. R., Acharya, S. and Rao, J. C. (2020). School Interventions-based Prevention of Early-Childhood Caries among 3-5-year-old children from very low socioeconomic status: Two-year randomized trial. Journal of public health dentistry, 80(1), pp. 51-60.

Sathish, T. and Karthick, S. (2020). Wear behaviour analysis on aluminium alloy 7050 with reinforced SiC through taguchi approach. Journal of Japan Research Institute for Advanced Copper-Base Materials and Technologies, 9(3), pp. 3481-3487.

Sharma, P. et al. (2019). Emerging trends in the novel drug delivery approaches for the treatment of lung cancer. Chemico-biological interactions, 309, p. 108720.

Singh, A. et al. (1962). Endemic Fluorosis: With Particular Reference to Dental and Systemic Intoxication, Postgraduate Medical Journal, pp. 150-156.

Singh, V. P., Yadav, S. and Yadava, R. N. (2018). Water Quality Management: Select Proceedings of ICWEES2016. Springer.

Sridharan, G. et al. (2019). Evaluation of salivary metabolomics in oral leukoplakia and oral squamous cell carcinoma. Journal of oral pathology \& medicine: official publication of the International Association of Oral Pathologists and the American Academy of Oral Pathology, 48(4), pp. 299-306.

Vandana, K. L. and Sesha Reddy, M. (2007). Assessment of periodontal status in dental fluorosis subjects using community periodontal index of treatment needs. Indian 
Journal of Dental Research, p. 67.

Varghese, S. et al. (2015). Estimation of salivary tumor necrosis factor-alpha in chronic and aggressive periodontitis patients. Contemporary Clinical Dentistry, p. 152.

Varghese, S. S., Ramesh, A. and Veeraiyan, D. N. (2019). Blended Module-Based Teaching in Biostatistics and Research Methodology: A Retrospective Study with Postgraduate Dental Students. Journal of dental education, 83(4), pp. 445-450.

Vignesh, R. et al. (2019). Management of Complicated
Crown-Root Fracture by Extra-Oral Fragment Reattachment and Intentional Reimplantation with 2 Years Review, Contemporary clinical dentistry, 10(2), pp. 397-401.

Vijayashree Priyadharsini, J. (2019). In silico validation of the non-antibiotic drugs acetaminophen and ibuprofen as antibacterial agents against red complex pathogens. Journal of periodontology, 90(12), pp. 1441-1448.

Zimmermann, E. R., Leone, N. C. and Arnold, F. A. (1955) Oral aspects of excessive fluorides in a water supply. The Journal of the American Dental Association, pp. 272-277. 